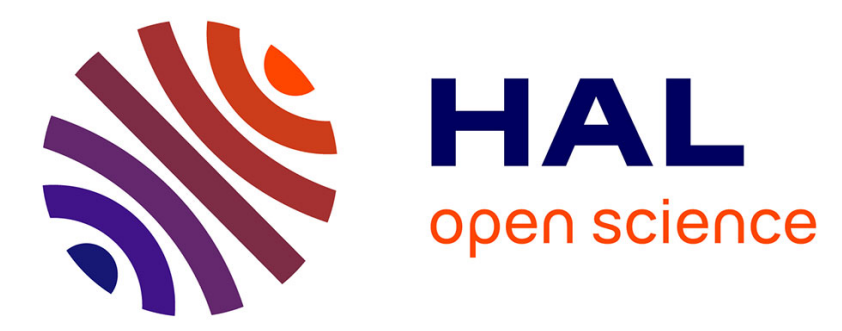

\title{
Comparison of time-to-positivity between two blood culture systems: a detailed analysis down to the genus-level
}

\author{
Rémi Le Guern, Marie Titécat, Caroline Loïez, Claire Duployez, Frédéric
} Wallet, Rodrigue Dessein

\section{To cite this version:}

Rémi Le Guern, Marie Titécat, Caroline Loïez, Claire Duployez, Frédéric Wallet, et al.. Comparison of time-to-positivity between two blood culture systems: a detailed analysis down to the genus-level. European Journal of Clinical Microbiology and Infectious Diseases, 2021, 10.1007/s10096-021-041759. hal-03240927

\section{HAL Id: hal-03240927 https://hal.science/hal-03240927}

Submitted on 28 May 2021

HAL is a multi-disciplinary open access archive for the deposit and dissemination of scientific research documents, whether they are published or not. The documents may come from teaching and research institutions in France or abroad, or from public or private research centers.
L'archive ouverte pluridisciplinaire HAL, est destinée au dépôt et à la diffusion de documents scientifiques de niveau recherche, publiés ou non, émanant des établissements d'enseignement et de recherche français ou étrangers, des laboratoires publics ou privés. 
1 Title: Comparison of time-to-positivity between two blood culture systems: a detailed 2 analysis down to the genus-level

3 Author names and affiliations: Rémi Le Guern ${ }^{1,2}$, Marie Titécat ${ }^{1,3}$, Caroline Loïez ${ }^{1}$, 4 Claire Duployez ${ }^{1,2}$, Frédéric Wallet ${ }^{1,2}$, Rodrigue Dessein ${ }^{1,2}$

$5{ }^{1}$ CHU Lille, Laboratoire de Bactériologie-Hygiène, F-59000 Lille, France.

$6 \quad{ }^{2}$ Univ. Lille, CNRS, Inserm, CHU Lille, Institut Pasteur Lille, U1019 - UMR 9017 - CIIL 7 Center for Infection and Immunity of Lille, F-59000 Lille, France.

$8{ }^{3}$ Univ. Lille, Inserm, CHU Lille, U1286 - INFINITE - Institute for Translational Research 9 in Inflammation, F-59000 Lille, France.

10 Corresponding author: Rémi Le Guern. Service de Bactériologie, Centre de Biologie 11 Pathologie Génétique. Boulevard du Professeur Jules Leclercq. 59037 Lille France.

12 Email address: remi.leguern@chru-lille.fr 


\section{Abstract}

A recently developed, automated blood culture system and medium improve the timeto-positivity (TTP) for bacteremia. However, there have thus far been no genus-level analyses using this novel system. We evaluated and compared the changes in blood culture TTP between two systems: BacT/Alert 3D with a blood culture medium containing activated charcoal versus the more recent BacT/Alert Virtuo with a blood culture medium containing polymeric beads. This before-and-after study included blood cultures collected between July 2010 and April 2014 (3D, activated charcoal) and between July 2015 and April 2018 (Virtuo, polymeric beads). A total of 554,732 blood cultures were included, 267,935 (48.30\%) during the first period and 286,797 (51.70\%) during the second period. Overall, 55,611 (10.02\%) tested positive for at least one microorganism. The incubation of the blood culture medium in the Virtuo system was associated with reduced TTP for the most prevalent bacteria, those representing $91.72 \%(n=51,006)$ of all the positive blood cultures. The median TTP was reduced by 0.99 hours for Staphylococcus, Enterococcus, Streptococcus, Pseudomonadales, and most of the genera within the order Enterobacterales (except the family Morganellaceae). However, strictly anaerobic bacteria belonging to the genus Bacteroides, representing $0.85 \%(n=474)$ of all positive blood cultures, were detected 4.53 hours later using the Virtuo system. Virtuo was associated with a shorter TTP for most bacteria, but this improvement was heterogeneous to the genus level.

Keywords: Bacteriological Techniques; Bacteremia; Bloodstream Infection; Bacteroides Infection; Time-to-positivity 


\section{Introduction}

37 Bloodstream infections (BSI) are frequent and severe systemic infections associated with a 30 -day mortality rate of $13-21 \%$ [1]. Empiric antimicrobial therapy is usually prescribed but is not always appropriate and should be re-evaluated depending on

40 bacteriological analyses. Thus, fast and accurate pathogen diagnostics lead to better

41 care of patients with BSI [2].

42 Recent blood culture automated systems have been developed to optimize BSI

43 pathogen detection by improving the broth composition of the blood culture bottles and

44 the monitoring system. The BacT/Alert Virtuo (bioMérieux, Marcy l'Etoile, France;

45 referred to as Virtuo hereafter) automated system presents improved temperature 46 stability and high-fidelity optics compared to the older version BacT/Alert 3D

47 (bioMérieux, Marcy l'Etoile, France; referred to as 3D hereafter) [3]. The Virtuo is

48 intended to be used with a blood culture medium containing antibiotic-binding polymeric

49 beads instead of the former blood culture medium containing activated charcoal [4]. The

50 broth composition of blood cultures with activated charcoal is based on soybean-casein

51 digest with brain heart infusions solids. whereas for blood cultures with polymeric beads

52 the broth composition is based on peptones and yeast extracts without brain heart

53 infusions solids [5]. Polymeric beads provide a cleaner background for Gram-staining

54 and less interference for MALDI-TOF mass spectrometry or molecular assays, 55 compared to activated charcoal.

56 Time-to-positivity (TTP) of blood cultures is an important parameter to optimize because

57 lower TTP leads to faster pathogen identification, guiding the antimicrobial therapy. 
58 Utilization of the Virtuo with blood culture media containing polymeric beads reduced 59 the time-to-positivity (TTP) of blood cultures for Enterobacterales and Enterococcus 60 spp., and increased bacterial recovery [3, 4, 6]. However, a detailed genus-level 61 analysis was not feasible in this type of prospective studies because the number of

62 blood culture bottles required would have been prohibitively large. Indeed, only about $6310 \%$ of the blood culture samples are positive, and most of the bacteria recovered 64 belong to the same genera (Staphylococcus, Escherichia, Enterococcus, or 65 Streptococcus) [7]. Thus, the impact of switching to a newer automated system and 66 blood culture medium on the detection of less prevalent bacteria remains unknown.

67 We performed a large before-and-after study to examine the impact of switching to a 68 modern automated system and blood culture medium. The principal aim was to 69 evaluate the changes in TTP values, detailed to the genus level, between two systems: 3D with a blood culture medium containing activated charcoal versus the more recent

71 Virtuo with a blood culture medium containing polymeric beads.

\section{Methods}

73 This before-and-after study was conducted at Lille University Hospital, a 3,200-bed 74 hospital in France. In the first period from July 2010 to April 2014, BacT/Alert FA 75 (aerobic), FN (anaerobic), or PF (pediatric) blood cultures (containing activated 76 charcoal) were incubated on a 3D automated blood culture system. In the second period 77 from July 2015 to April 2018, BacT/Alert FA Plus (aerobic), FN Plus (anaerobic), or PF 78 Plus (pediatrics) blood cultures (containing polymeric beads) were incubated on a 79 Virtuo. In both periods, bottles were incubated for five days or until they signaled 
positive for growth. Blood cultures from May 2014 to June 2015 were excluded from the

81 analysis because they were collected within a transition period during which another

82 blood culture medium (containing polymeric beads) was used on a 3D.

83 Blood cultures were transferred from the clinical wards to the laboratory using a 84 pneumatic tube system. Positive blood cultures were processed according to standard 85 laboratory protocols. Bacteria were identified using MALDI-TOF mass spectrometry 86 (Bruker Daltonics, Wissembourg, France). If identification at the species level was not 87 possible using MALDI-TOF, 16S rDNA sequencing was performed, as described 88 elsewhere [8].

Data collected included the type of blood culture, time of specimen collection, time of 90 introduction into the automated blood culture system, and time of positivity or negativity.

91 Time-to-positivity was defined as the duration from when the sample was first incubated 92 on the automated blood culture system to signaled positive for growth by the system. 93 The following patient demographic data were automatically collected during the data 94 extraction: age, sex, ward, and date of entry into the hospital. No names or birthdates were included to preserve the privacy of patients, following the recommendations from 96 the French National Commission on Informatics and Liberty.

97 Polymicrobial blood cultures were not excluded. If a blood culture was positive with 98 multiple species from the same genus (e.g., two different Staphylococcus isolates), it 99 100 was counted only once for this genus. The denominator was the total number of blood culture bottles included and not the number of different bacteria identified. 
101 Statistical analyses were performed using R software version 3.6.3 [9]. The statistical 102 unit was blood culture bottles, which were considered individually for the analysis, even 103 if they were sampled as a set. Bacterial names were automatically classified by the 104 AMR R package [10], using the microbial taxonomy from the Catalogue of Life 105 database. Failures or uncertainties in the classification were manually reviewed. 106 Qualitative variables were compared with Fisher's exact test and continuous variables 107 with the Mann-Whitney $U$ test. Survival distributions, as defined by the delay until blood 108 cultures signaled positive for growth, were plotted using the survminer R package. 


\section{Results}

110 A total of 554,732 blood cultures were included. Of these, $267,935(48.30 \%)$ were in the

111 charcoal-containing blood culture medium incubated on a 3D, and $286,797(51.70 \%)$

112 were in the polymeric bead-containing blood culture medium incubated on a Virtuo. In

113 total, 55,611 blood cultures (10.02\%) were positive for at least one microorganism,

114 including $1,514(0.27 \%)$ with yeasts. Polymicrobial growth (blood culture positive with

115 multiple microorganisms) was detected in 3,613 (0.65\%) blood cultures.

116 The characteristics of the analyzed blood cultures are provided in Table 1. Samples 117 were collected mainly from patients hospitalized in the medical $(35.17 \%)$, intensive or

118 critical care $(33.88 \%)$, surgical $(11.08 \%)$, emergency $(8.73 \%)$, and pediatric $(5.03 \%)$

119 wards. There were small but statistically significant differences in the following

120 demographic data between the two populations: patients from the Virtuo group were

121 older (58 vs. 60 years), more frequently female $(39.03 \%$ vs. $40.00 \%)$, and more

122 frequently from emergency wards $(6.65 \%$ vs. $10.66 \%)$. The blood culture TTP was

123 lower for the Virtuo (16.53 hours on 3D vs. 15.40 hours on Virtuo, $P<0.0001$ ). In

124 subgroup analysis, the blood culture TTP was reduced for aerobic blood bottles (15.98

125 hours on 3D vs. 14.75 hours on Virtuo, $P<0.0001)$, for anaerobic blood bottles (17.07

126 hours vs. 16.65 hours, $P<0.0001$ ), and for pediatric blood bottles (17.88 hours vs.

12715.28 hours, $P<0.0001)$.

128 For Gram-positive bacteria, we compared the most frequent genera, identified in at least

129100 blood cultures (Table 2). Staphylococcus represented the most prevalent genus,

130 identified in $53.05 \%$ of the positive blood cultures, with a lower TTP for the Virtuo. 
131 Enterococcus and Streptococcus were present in $6.69 \%$ and $5.45 \%$ of the positive 132 blood cultures, respectively, also with lower TTP in the second period. The TTP was 133 higher in the Virtuo for Micrococcus, which are usually considered contaminating 134 bacteria with little clinical relevance. Table S1 shows the subgroup analysis for Gram135 positive bacteria, depending on the type of blood culture bottles.

136 For Gram-negative bacteria, we compared the most frequent genera, identified in at 137 least 100 blood cultures (Table 3). Enterobacterales represented the most frequent 138 order, identified in $28.11 \%$ of the positive blood cultures. Of these, the TTP for the

139 Virtuo was lower for genera belonging to the family Enterobacteriaceae (Escherichia, 140 Klebsiella, Enterobacter, Citrobacter) or Yersiniaceae (Serratia). However, there were 141 no differences in the detection of two genera belonging to the family Morganellaceae 142 (Proteus and Morganella) despite high numbers of positive blood cultures ( $n=556$ and $143 n=267$, respectively). Pseudomonas was identified in $3.20 \%$ of the positive blood 144 cultures, and the TTP was lower for the Virtuo. Bacteroides were the most frequently 145 strictly anaerobic bacteria identified $(0.85 \%$ of the positive blood cultures) and had a 146 higher TTP for the Virtuo (26.62 hours on 3D vs. 31.15 on Virtuo, $P<0.0001$ ). The TTP 147 values of blood cultures positive with Bacteroides were plotted as cumulative events in 148 Figure 1. Table S2 shows the subgroup analysis for Gram-negative bacteria, depending 149 on the type of blood culture bottles.

150 For yeasts, the TTP tended to be lower in the Virtuo, but this was not statistically 151 significant (31.39 vs. 33.50 hours, $P=0.056)$.

\section{Discussion}


153 In this before-and-after study on 554,732 blood cultures, incubation with the system 154 combining the Virtuo and polymeric beads was associated with a reduced TTP for the 155 most prevalent bacteria. The median TTP was reduced by 0.99 hours for 156 Staphylococcus, Enterococcus, Streptococcus, Pseudomonadales, and most of the 157 genera within the order Enterobacterales (except the family Morganellaceae), 158 representing $91.72 \%(n=51,006)$ of all the positive blood cultures.

159 These results are consistent with previous studies. Altun et al. showed that the TTP was 160 significantly reduced in the Virtuo compared with the 3D using simulated blood cultures 161 spiked with 115 bacterial or fungal isolates, for all the bacteria tested (Enterobacterales, 162 Acinetobacter, Staphylococcus, Enterococcus, Streptococcus) [3]. The TTP was 163 reduced in the Virtuo for both blood culture media, containing either polymeric beads 164 (FA Plus or FN Plus) or activated charcoal (FA or FN). Another study using simulated 165 blood cultures spiked with 330 bacterial or fungal isolates showed a reduction of the 166 TTP in the Virtuo for most microorganisms, except Stenotrophomonas maltophilia and 167 some Candida species [11]. Additionally, a multicenter study on 5,709 blood culture sets 168 compared the 3D to the Virtuo using either a blood culture medium containing polymeric 169 beads (FA Plus or FN Plus) or a medium containing no beads and no charcoal (SA or $170 \mathrm{SN})$ [6]. In these conditions, the TTP was reduced by almost 2 hours for the blood 171 cultures incubated on the Virtuo compared to the 3D. The TTP was significantly 172 decreased only for Enterobacterales and Enterococcus. For Streptococcus and 173 coagulase-negative Staphylococcus, there was a trend of the Virtuo reducing the TTP, 174 but this effect was not statistically significant $(P=0.08$ and $P=0.10$, respectively). A 175 recent study compared the TTP of the 3D to Virtuo on 1,904 blood culture sets 
176 exclusively using a blood culture medium containing polymeric beads (FA Plus or FN 177 Plus) [12]. The TTP was significantly reduced for Staphylococcus aureus ( -1.7 hours for 178 the Virtuo) and Escherichia coli (-0.6 hours). However, the overall reduction of the TTP 179 for clinically significant pathogens was only 18 minutes, less than the 2-hour reduction 180 reported previously [6]. Finally, another study compared two different periods, one with 181 the 3D, followed by another with the Virtuo, exclusively using a blood culture medium 182 containing polymeric beads (FA Plus, FN Plus, or PF Plus) [13]. The TTP was 183 significantly reduced for Staphylococcus, Enterobacterales, and Streptococcus, while no 184 difference was shown for Enterococcus.

185 In our study, the TTP was reduced in the Virtuo with polymeric beads for 186 Enterobacterales, but not for the Morganellaceae family. Data on bacteremia due to 187 Morganellaceae are scarce [14], even if Morganellaceae represented $1.50 \%$ of all 188 positive blood cultures in our study. The mechanism by which the Morganellaceae TTP 189 is not decreased with the more efficient automated system remains unknown. One 190 hypothesis might reside in activated charcoal inhibiting swarming from Proteus [15]. A 191 correlation between swarming and growth rate in liquid medium has been previously 192 suggested [16].

193 Our results also show that Bacteroides was associated with a higher TTP for the Virtuo 194 with polymeric beads compared with the 3D with activated charcoal. In 474 blood 195 culture positive with Bacteroides, the median TTP was 4.53 hours higher using the 196 Virtuo $(P<0.0001)$. Bacteroides is the most common genus detected in BSI due to 197 strictly anaerobic bacteria. This delayed detection could be clinically relevant. Failure to 198 add appropriate antimicrobials was previously associated with higher mortality (55\% vs. 
199

200

201

202

203

204

205

206

207

208

209

210

211

212

213

214

215

216

217

218

219

220

221

$17 \%$ ) in the case of anaerobic bacteremia (mostly due to Bacteroides) [17]. Empirical antimicrobial therapy includes anti-anaerobic coverage for only half of the patients with anaerobic bacteremia [17]. The reasons for this delayed detection remain hypothetic. As the Virtuo improved temperature stability and high-fidelity optics compared to the 3D, the difference in TTP for Bacteroides might be linked to the use of a blood culture medium containing polymeric beads. Indeed, De Keukeleire et al. compared the performance of two blood culture media containing either polymeric beads (FN plus) or activated charcoal (FN) on the detection of anaerobes with the $3 \mathrm{D}$, using blood cultures experimentally spiked with anaerobic bacteria [18]. The TTP was significantly increased for resin-based compared with charcoal-based blood culture bottles for Bacteroides spp. (49 hours vs. 27 hours) and most of the other anaerobes. Rapid diagnosis of bacteremia due to Bacteroides would allow for the rapid adaptation of antimicrobial therapy, which may lead to lower mortality.

The principal limitation of our study is the before-and-after design. First, the type of patients included in our hospital may have changed during the study period. Demographic characteristics of the patients were significantly different: in the first period, patients were younger (58 vs. 60 years), more frequently male $(60.97 \%$ vs. $60.00 \%)$, and more frequently hospitalized in intensive or critical care $(34.59 \%$ vs. $33.22 \%)$. All these differences were highly significant $(P<0.0001)$. However, due to a large number of blood cultures included $(554,732)$, even small differences in values could result in statistical significance. Second, additional parameters affecting the TTP of blood cultures include the volume of blood sampled, transportation time, and concomitant antimicrobial therapy, which have not been monitored in our study [19]. 
222 Finally, different blood culture media and blood culture instruments were compared, 223 resulting in a mixed effect. However, the TTP decrease for the most prevalent bacteria 224 found is consistent with the literature $[3,6,13]$ and externally validate our results.

225 Overall, our study confirms the Virtuo and the blood culture medium with polymeric 226 beads are associated with a reduced TTP for the most prevalent bacteria, compared to 227 the 3D system and the blood culture medium with activated charcoal. However, no 228 differences were observed for the family Morganellaceae, representing an exception 229 within the order Enterobacterales. Moreover, TTP values were even significantly 230 increased for Bacteroides using the most modern automated system. 


\section{Declarations}

232 Funding: This work was supported by CHU Lille.

233 Competing Interests: The authors declare that they have no conflict of interest.

234 Ethical Approval: The study was approved by the local Institutional Review Board. 235 Patient consent was not requested for this study, since the study was based on 236 anonymized data collected retrospectively.

237 Consent to Participate: Formal consents were not required for this retrospective study 238 on anonymized data.

239 Consent to Publish: Not applicable.

240 Availability of data and materials: All data were anonymized. The dataset analyzed 241 during the current study is available from the corresponding author on reasonable 242 request.

243 Authors Contributions: RLG and RD conceived and planned the study. All authors 244 participated in data collection and microbiological analysis. RLG performed the data 245 analysis. RLG and RD wrote the first draft, all authors reviewed and edited the final 246 manuscript. 
248 Words: 2289

249 Figure Legends

250 Figure 1. Cumulative probability of the time-to-positivity (TTP) for blood cultures 251 positive with Bacteroides sp.

252 BacT/Alert 3D = BacT/Alert 3D with the blood culture medium containing activated 253 charcoal; BacT/Alert Virtuo = BacT/Alert Virtuo with the blood culture medium containing 254 polymeric beads. 
257 [1] Goto M, Al-Hasan MN (2013) Overall burden of bloodstream infection and

258 nosocomial bloodstream infection in North America and Europe. Clin Microbiol Infect 19

$259 \quad(6): 501-509$

260 [2] Lamy B, Sundqvist M, Idelevich EA, Escmid Study Group for Bloodstream

261 Infections E, Sepsis (2020) Bloodstream infections - Standard and progress in pathogen

262 diagnostics. Clin Microbiol Infect 26 (2):142-150

263 [3] Altun O, Almuhayawi M, Luthje P, Taha R, Ullberg M, Ozenci V (2016) Controlled

264 Evaluation of the New BacT/Alert Virtuo Blood Culture System for Detection and Time

265 to Detection of Bacteria and Yeasts. J Clin Microbiol 54 (4):1148-1151

266 [4] Kirn TJ, Mirrett S, Reller LB, Weinstein MP (2014) Controlled clinical comparison

267 of BacT/alert FA plus and FN plus blood culture media with BacT/alert FA and FN blood 268 culture media. J Clin Microbiol 52 (3):839-843

269 [5] Dunne WM, Burnham C-AD (2018) The dark art of blood cultures. ASM Press, 270 Washington, DC

271 [6] Jacobs MR, Mazzulli T, Hazen KC, Good CE, Abdelhamed AM, Lo P, Shum B, 272 Roman KP, Robinson DC (2017) Multicenter Clinical Evaluation of BacT/Alert Virtuo 273 Blood Culture System. J Clin Microbiol 55 (8):2413-2421

274 [7] Weinstein MP, Towns ML, Quartey SM, Mirrett S, Reimer LG, Parmigiani G, 275 Reller LB (1997) The clinical significance of positive blood cultures in the 1990s: a 276 prospective comprehensive evaluation of the microbiology, epidemiology, and outcome 277 of bacteremia and fungemia in adults. Clin Infect Dis 24 (4):584-602 
278 [8] Le Guern R, Loiez C, Armand S, Marceau L, Courcol R, Wallet F (2015) Infective 279 endocarditis: does a new 16 S rDNA set of primers improve the microbiological 280 diagnosis? Infect Dis (Lond) 47 (12):896-901

281 [9] R Core Team (2013) R: A language and environment for statistical computing.

282 [10] Berends MS, Luz CF, Friedrich AW, Sinha BNM, Albers CJ, Glasner C (2019)

283 AMR - An R Package for Working with Antimicrobial Resistance Data. bioRxiv:810622

284 [11] Menchinelli G, Liotti FM, Fiori B, De Angelis G, D'Inzeo T, Giordano L, Posteraro

285 B, Sabbatucci M, Sanguinetti M, Spanu T (2019) In vitro Evaluation of BACT/ALERT(R)

286 VIRTUO(R), BACT/ALERT 3D(R), and BACTEC FX Automated Blood Culture Systems

287 for Detection of Microbial Pathogens Using Simulated Human Blood Samples. Front

288 Microbiol 10:221

289 [12] Kim SC, Lee S, Kim S, Cho OH, Park H, Yu SM (2019) Comparison of Clinical

290 Performance Between BacT/Alert Virtuo and BacT/Alert 3D Blood Culture Systems. Ann

291 Lab Med $39(3): 278-283$

292 [13] Congestri F, Pedna MF, Fantini M, Samuelli M, Schiavone P, Torri A, Bertini S, 293 Sambri V (2017) Comparison of 'time to detection' values between BacT/ALERT

294 VIRTUO and BacT/ALERT 3D instruments for clinical blood culture samples. Int J Infect 295 Dis 62:1-5

296 [14] Kim BN, Kim NJ, Kim MN, Kim YS, Woo JH, Ryu J (2003) Bacteraemia due to 297 tribe Proteeae: a review of 132 cases during a decade (1991-2000). Scand J Infect Dis $298 \quad 35(2): 98-103$

299 [15] Hernandez E, Ramisse F, Cavalho JD (1999) Abolition of swarming of Proteus. J $300 \quad$ Clin Microbiol 37 (10):3435 
301 [16] Jones HE, Park RW (1967) The influence of medium composition on the growth 302 and swarming of Proteus. J Gen Microbiol 47 (3):369-378

303 [17] Salonen JH, Eerola E, Meurman O (1998) Clinical significance and outcome of 304 anaerobic bacteremia. Clin Infect Dis 26 (6):1413-1417

305 [18] De Keukeleire S, Wybo I, Emmerechts K, Pierard D (2015) Performance of 306 BacT/Alert resin-based FN plus bottles compared with BacT/Alert charcoal-based FN 307 bottles for the detection of anaerobes in experimentally seeded blood cultures.

308 Anaerobe 35 (Pt B):92-95

309 [19] Lamy B (2019) Blood culture time-to-positivity: making use of the hidden 310 information. Clin Microbiol Infect 25 (3):268-271 
312 Table 1. Characteristics of the included blood culture sample bottles

\begin{tabular}{|c|c|c|c|}
\hline & $\begin{array}{c}\text { BacT/Alert 3D } \\
\text { Total }(n=267,935)\end{array}$ & $\begin{array}{c}\text { BacT/Alert Virtuo } \\
\text { Total }(n=286,797)\end{array}$ & $P$-value \\
\hline \multicolumn{4}{|c|}{ Patient demographics characteristics } \\
\hline Age (years) & $58.0(42.0-70.0)$ & $60.0(45.0-71.0)$ & $<0.0001$ \\
\hline Sex & & & $<0.0001$ \\
\hline Female & $104,554(39.03 \%)$ & $114,705(40.00 \%)$ & .. \\
\hline Male & $163,355(60.97 \%)$ & $172,085(60.00 \%)$ & .. \\
\hline Type of ward & & & $<0.0001$ \\
\hline Medicine & $94,750(35.36 \%)$ & $100,367(35.00 \%)$ & .. \\
\hline Intensive/critical care & $92,676(34.59 \%)$ & $95,273(33.22 \%)$ & .. \\
\hline Surgery & $30,592(11.42 \%)$ & $30,851(10.76 \%)$ & .. \\
\hline Emergency & $17,825(6.65 \%)$ & $30,583(10.66 \%)$ & .. \\
\hline Pediatric & $15,362(5.73 \%)$ & $12,530(4.37 \%)$ & .. \\
\hline Geriatric & $9,870(3.69 \%)$ & $9,511(3.31 \%)$ & .. \\
\hline Rehabilitation & $3,740(1.40 \%)$ & $4,983(1.74 \%)$ & .. \\
\hline Obstetrics & $2,958(1.10 \%)$ & $2,576(0.90 \%)$ & .. \\
\hline Psychiatry & $162(0.06 \%)$ & $123(0.04 \%)$ & .. \\
\hline Blood culture type & & & $<0.0001$ \\
\hline Aerobic & $130,598(48.74 \%)$ & $141,543(49.35 \%)$ &.. \\
\hline Anaerobic & 118,757 (44.32\%) & $130,893(45.64 \%)$ & .. \\
\hline Pediatric & $18,580(6.94 \%)$ & $14,361(5.00 \%)$ & .. \\
\hline \multicolumn{4}{|l|}{ Positive blood cultures } \\
\hline Positive blood bottles & $25,783(9.62 \%)$ & $29,828(10.40 \%)$ & $<0.0001$ \\
\hline $\begin{array}{l}\text { Time to positivity } \\
\text { (hours) }\end{array}$ & $16.53(11.88-24.63)$ & $15.40(10.95-22.72)$ & $<0.0001$ \\
\hline
\end{tabular}

Data

are

median

(IQR)

or

$\mathrm{n}$

(\%). 
Table 2. Gram-positive bacteria: time-to-positivity (median) and percentage of positivity

\begin{tabular}{|c|c|c|c|c|c|c|c|c|c|c|c|}
\hline \multicolumn{3}{|c|}{ Microorganism group } & \multicolumn{2}{|c|}{ Sample size $(n)$} & \multicolumn{4}{|c|}{ Median time to positivity (hours) } & \multicolumn{3}{|c|}{$\begin{array}{c}\text { Percentage of positivity } \\
(\%)\end{array}$} \\
\hline Phylum & Order & Genus & $3 \mathrm{D}$ & Virtuo & $3 \mathrm{D}$ & Virtuo & Difference & $P$-value & $3 \mathrm{D}$ & Virtuo & $P$-value \\
\hline \multicolumn{3}{|c|}{ Firmicutes } & 16,716 & 19,385 & & & & & & & \\
\hline \multicolumn{3}{|c|}{ Bacillales } & 13,625 & 16,123 & & & & & & & \\
\hline & & Staphylococcus & 13,520 & 15,984 & 19.27 & 18.35 & -0.92 & $<0.0001$ & 5.05 & 5.57 & $<0.0001$ \\
\hline & & Bacillus & 79 & 143 & 12.32 & 12.9 & 0.58 & 0.92 & 0.03 & 0.05 & $<0.001$ \\
\hline \multicolumn{3}{|c|}{ Lactobacillales } & 3,340 & 3,512 & & & & & & & \\
\hline & & Enterococcus & 1,789 & 1,931 & 13.05 & 12.50 & -0.55 & $<0.001$ & 0.67 & 0.67 & 0.81 \\
\hline & & Streptococcus & 1,506 & 1,526 & 13.30 & 12.46 & -0.84 & $<0.0001$ & 0.56 & 0.53 & 0.13 \\
\hline \multicolumn{3}{|c|}{ Actinobacteria } & 291 & 386 & & & & & & & \\
\hline \multicolumn{3}{|c|}{ Actinomycetales } & 262 & 349 & & & & & & & \\
\hline & & Micrococcus & 101 & 145 & 37.02 & 43.68 & 6.66 & $<0.001$ & 0.04 & 0.05 & 0.03 \\
\hline & & Corynebacterium & 86 & 73 & 47.05 & 49.58 & 2.53 & 0.59 & 0.03 & 0.03 & 0.17 \\
\hline
\end{tabular}

Only genera identified in at least 100 blood cultures are shown. Statistically significant differences in time-to-positivity (TTP) are in bold. 3D = BacT/Alert 3D with the blood culture medium containing activated charcoal; Virtuo = BacT/Alert Virtuo with blood culture medium containing polymeric beads. 
Table 3. Gram-negative bacteria: time-to-positivity (median) and percentage of positivity

\begin{tabular}{|c|c|c|c|c|c|c|c|c|c|c|c|}
\hline \multicolumn{3}{|c|}{ Microorganism group } & \multicolumn{2}{|c|}{ Sample size $(n)$} & \multicolumn{4}{|c|}{ Median time to positivity (hours) } & \multicolumn{3}{|c|}{$\begin{array}{l}\text { Percentage of positivity } \\
(\%)\end{array}$} \\
\hline Phylum & Order & Genus & $3 D$ & Virtuo & 3D & Virtuo & Difference & $P$ value & $3 \mathrm{D}$ & Virtuo & $P$-value \\
\hline \multicolumn{3}{|c|}{ Proteobacteria } & 8,248 & 10,001 & & & & & & & \\
\hline \multicolumn{3}{|c|}{ Enterobacterales } & 6,970 & 8,660 & & & & & & & \\
\hline & & Escherichia & 3,197 & 3,791 & 11.08 & 10.30 & -0.78 & $<0.0001$ & 1.19 & 1.32 & $<0.0001$ \\
\hline & & Klebsiella & 1,847 & 2,367 & 11.57 & 10.28 & -1.29 & $<0.0001$ & 0.69 & 0.83 & $<0.0001$ \\
\hline & & Enterobacter & 1,170 & 1,581 & 12.07 & 10.77 & -1.30 & $<0.0001$ & 0.44 & 0.55 & $<0.0001$ \\
\hline & & Serratia & 240 & 319 & 14.43 & 12.32 & -2.11 & $<0.0001$ & 0.09 & 0.11 & 0.01 \\
\hline & & Proteus & 275 & 281 & 12.73 & 13.15 & 0.42 & 0.84 & 0.10 & 0.10 & 0.61 \\
\hline & & Citrobacter & 189 & 318 & 11.73 & 10.76 & -0.97 & $<0.001$ & 0.07 & 0.11 & $<0.0001$ \\
\hline & & Morganella & 119 & 148 & 13.32 & 12.82 & -0.50 & 0.37 & 0.04 & 0.05 & 0.25 \\
\hline \multicolumn{3}{|c|}{ Pseudomonadales } & 1,072 & 1,092 & & & & & & & \\
\hline & & Pseudomonas & 922 & 859 & 17.23 & 16.35 & -0.88 & 0.03 & 0.34 & 0.30 & 0.004 \\
\hline & & Acinetobacter & 138 & 215 & 13.16 & 11.80 & -1.36 & 0.04 & 0.05 & 0.08 & $<0.001$ \\
\hline \multicolumn{3}{|c|}{ Xanthomonadales } & 83 & 119 & & & & & & & \\
\hline & & Stenotrophomonas & 83 & 119 & 22.58 & 20.62 & -1.96 & 0.23 & 0.03 & 0.04 & 0.05 \\
\hline \multicolumn{3}{|c|}{ Bacteroidetes } & 298 & 256 & & & & & & & \\
\hline \multicolumn{3}{|c|}{ Bacteroidales } & 277 & 226 & & & & & & & \\
\hline & & Bacteroides & 261 & 213 & 26.62 & 31.15 & 4.53 & $<0.0001$ & 0.10 & 0.07 & 0.004 \\
\hline
\end{tabular}

Only genera identified in at least 100 blood cultures are shown. Statistically significant differences in time-to-positivity (TTP) are in bold. 3D = BacT/Alert 3D with the blood culture medium containing activated charcoal; Virtuo = BacT/Alert Virtuo with the blood culture medium containing polymeric beads. 


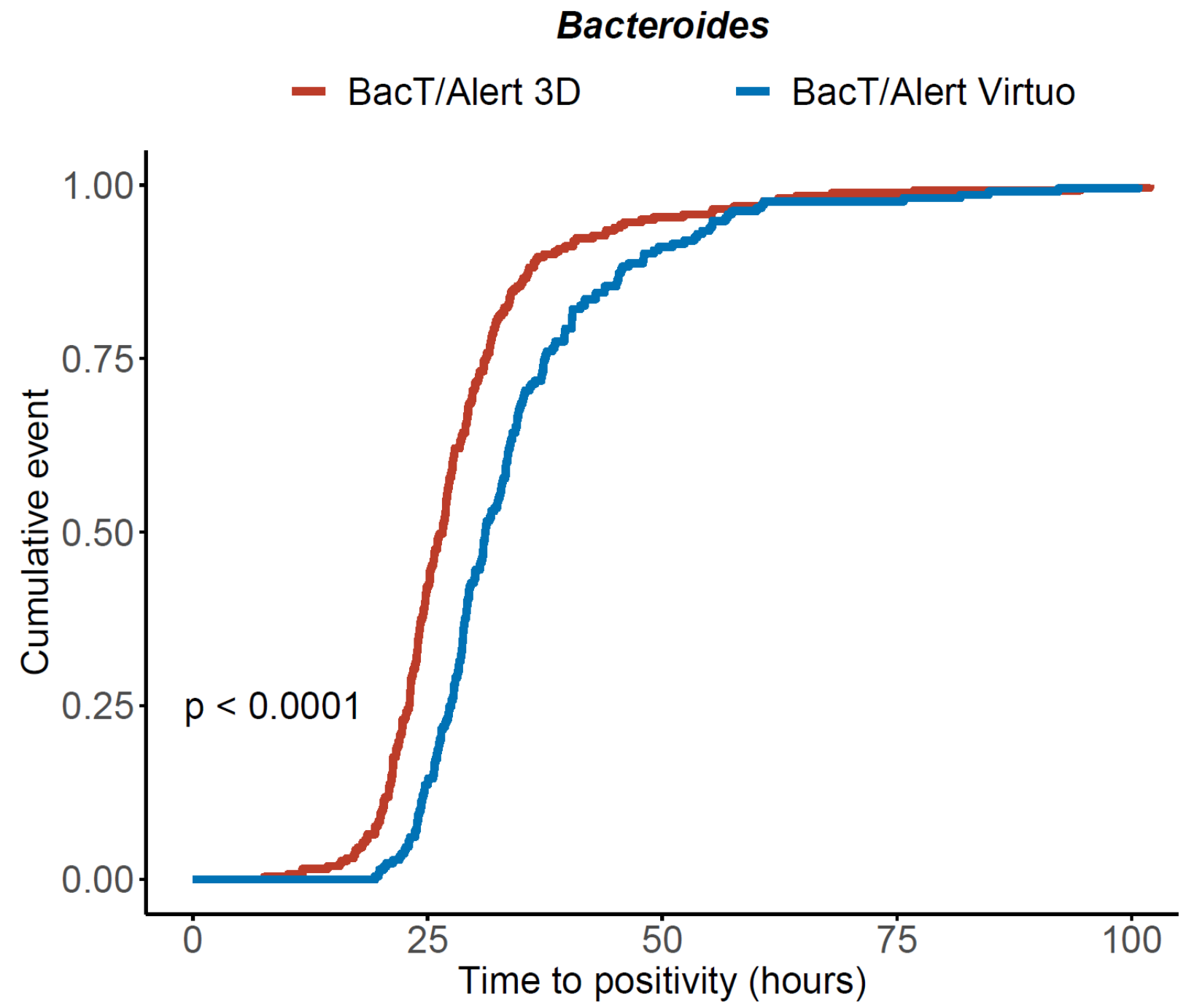

Figure 1. Cumulative probability of time to positivity for blood cultures positive with Bacteroides sp.

BacT/Alert 3D = BacT/Alert 3D with the blood culture medium containing activated charcoal; BacT/Alert Virtuo $=$ BacT/Alert Virtuo with the blood culture medium containing polymeric beads. 